\title{
Ultrasound guidelines for pediatric thyroid nodules: proceeding with caution
}

\author{
Jennifer E. Lim-Dunham ${ }^{1}$ \\ Received: 6 March 2019 / Accepted: 19 March 2019 / Published online: 1 June 2019 \\ (C) Springer-Verlag GmbH Germany, part of Springer Nature 2019
}

As pediatric radiologists, we encounter thyroid nodules on ultrasounds in children with increasing regularity in daily practice. We are often puzzled by how to approach these lesions with regard to describing their appearance, indicating the significance of their ultrasound features, and suggesting management recommendations. The article by ShapiraZaltsberg et al. [1] in the current issue of Pediatric Radiology is an important addition to the literature that offers guidance for clinical decision-making.

Utilizing two sets of thyroid imaging, reporting and data systems (TI-RADS) developed for malignancy risk classification of nodules based on ultrasound in adults, the authors applied the criteria to a group of 75 children. They found that both of these guidelines performed reasonably well for diagnostic accuracy, with areas under the receiver operating characteristic (ROC) curve for malignancy risk of 0.74 and 0.72 for the 2011 Kwak and 2017 American College of Radiology (ACR) TI-RADS, respectively [2, 3]. Both scoring systems stratify nodules from categories 1 to 5 , with points allocated according to the number of suspicious ultrasound characteristics, and with each feature given equal weight in the Kwak version but differential weighting in the ACR version. In both, higher-number TI-RADS categories correspond to higher calculated risk of malignancy.

These results corroborate other studies showing that the ACR TI-RADS, already validated in large adult populations, holds potential for use in children for identifying nodules that are at high risk for malignancy and that therefore warrant fineneedle aspiration biopsy [4, 5]. A study of the ACR TI-RADS in 74 nodules in children was found to discriminate between

Jennifer E. Lim-Dunham

jlim2@lumc.edu

1 Department of Radiology,

Loyola University Chicago Stritch School of Medicine,

2160 S. First Ave., Maywood, IL 60153, USA malignant and benign, demonstrating that for every one-unit increase in TI-RADS category the likelihood of malignancy increased 2.73 times, and showing an area under the ROC curve of 0.75 [6].

Other popular thyroid nodule ultrasound risk stratification guidelines that utilize qualitative pattern recognition, rather than the quantitative point allocation of the ACR and Kwak TI-RADS, have also demonstrated promise in children. One such system is the 2015 American Thyroid Association (ATA) management guideline for children with thyroid nodules and differentiated thyroid cancer. This was developed specifically for the pediatric population, and it borrows its ultrasound criteria from the 2015 ATA management guideline for adults with thyroid nodules, on the assumption that concerning ultrasound features are similar between children and adults [7, 8]. In this system, multiple ultrasound features are grouped to form a composite constellation of characteristics to stratify nodules into five categories ranging from benign to high suspicion. Studies of the ATA criteria in children have shown positive and negative predictive values of $68 \%$ and $87 \%$, respectively, in 123 nodules, and sensitivity and specificity of $91 \%$ and $54 \%$, respectively, in 125 nodules [ 9,10$]$. Another study of 27 pediatric nodules showed that ATA level of suspicion was a significant predictor of malignancy, with an odds ratio of 8.5 [11].

While a growing body of evidence suggests that adult ultrasound guidelines could be expanded for use in children, it is worthwhile to consider a few points of caution before embracing these algorithms in their entirety. Because of the differing epidemiologic and biologic behavior of thyroid cancer in the pediatric versus adult populations, refinements to adapt adult protocols for children are advisable. In adults, there has been a prevailing movement toward a conservative approach in order to minimize potential harm from over-treatment of clinically insignificant cancers. In children, however, a more aggressive approach might be prudent because thyroid nodules in children are more likely to be malignant than in adults, making 
over-diagnosis less of a concern. Moreover, thyroid nodules are relatively less common in children than adults, reducing the risk of excessive burden on health care resources [7].

Although survival rates for pediatric thyroid cancers are generally excellent, children are more likely than adults to have metastatic disease to cervical lymph nodes at the time of diagnosis. Accordingly, the ATA guideline for children recommends that ultrasound evaluation of cervical lymph nodes be performed in all children with suspicious nodules [7]. While ACR TI-RADS acknowledges the importance of lymph nodes, it focuses primarily on the appearance of the thyroid nodule itself. For children, however, formally integrating distorted architecture, abnormal vasculature, microcalcifications and other concerning lymph node features into a TI-RADS structure might be germane.

Reducing the number of unnecessary fine-needle aspiration biopsies of benign nodules in adults is partially accomplished by using progressively larger nodule size thresholds for triggering fine-needle aspiration biopsies with decreasing levels of suspicion. For example, ACR TI-RADS recommends fineneedle aspiration biopsies only for nodules over $2.5 \mathrm{~cm}$, $1.5 \mathrm{~cm}$ and $1.0 \mathrm{~cm}$ for categories 3,4 and 5 (mildly, moderately and highly suspicious), respectively [3]. These size thresholds will likely need to be lowered or eliminated for children. The ATA guideline for children states that because thyroid volume changes with age, determination of malignancy risk in children should be based solely on ultrasound appearance without regard for nodule size, a finding supported by subsequent work [6,7]. Prospective studies of larger numbers of children are needed to determine what, if any, size thresholds are appropriate for ACR TI-RADS in pediatrics.

The current paper and others show that ACR TI-RADS performs well in identifying benign and lower-suspicion nodules in children, therefore suggesting that deferment of fineneedle aspiration biopsies in appropriately selected nodules might be a reasonable management option $[1,6]$. Adult guidelines would dictate no further action, not even follow-up ultrasound, for ACR TI-RADS 1 and 2 (benign and not suspicious) lesions of any size [3]. Recent studies, however, acknowledge a number of false-negatives of up to $10-13 \%$ in children using ACR and ATA criteria, and therefore we believe that more stringent standards should be applied to children $[1,6,9,10]$. For nodules that do not warrant fine-needle aspiration biopsies, our own inclination is to perform surveillance follow-up ultrasounds. ACR TI-RADS advocates that categories 3, 4 and 5 nodules below the size threshold for fine-needle aspiration biopsies in adults undergo follow-up ultrasounds at intervals as frequently as once per year for up to 5 years, but additional study is required to determine what duration and intervals are optimal for children [3]. The ATA guideline for children recommends repeat ultrasound at 612 months, and every 1-2 years thereafter, even in nodules with proven benign cytology by fine-needle aspiration biopsies [7]. This suggests that the option of "no further evaluation" might have little role in children. Because of known early metastases to cervical lymph nodes, additional strategies could include ultrasound assessment of lateral neck compartments in all children with thyroid nodules, even nodules that are not particularly suspicious.

One consequence of more cautious management of pediatric nodules is an increase in the number of false-positives, leading to more fine-needle aspiration biopsies of benign nodules. The current paper indicates that $48 \%$ of benign nodules were classified as ACR TI-RADS 4 or 5 (moderately or highly suspicious), a finding supported by other studies $[1,6]$. This highlights the importance of critically appraising all features, especially those that exert a proportionately large influence on elevating the TI-RADS score. For example, the presence of punctate echogenic foci, a feature favoring malignancy because of its association with psammoma bodies in papillary thyroid carcinoma, is accorded 3 points [3]. The category of punctate echogenic foci, however, was found to be one for which inter-observer variability was highest, with this feature observed in the majority of benign as well as malignant pediatric nodules $[6,12]$. Our experience is similar to others that it is easy to overcall punctate echogenic foci because they can be difficult to distinguish from echogenicity related to intrinsic tissue heterogeneity and walls of microcysts [13]. Further education of radiologists and use of adjunct tools such as artificial intelligence and ultrasound elastography could help mitigate these effects.

Which one of these ultrasound risk classification systems should be used is a matter of debate, and will likely depend on institutional culture, radiologist and referring physician preference, and geographic bias. Some practitioners, especially those with more experience in evaluating nodules, might prefer the qualitative ATA-style system for the speed and ease with which it can be used. Up to $4 \%$ of nodules cannot be classified using such a pattern-based approach, however, and therefore others might prefer the quantitative point-based TIRADS style system which, while more cumbersome, can account for all nodules $[3,10]$. An important advantage of the ACR TI-RADS in particular is the structured lexicon using strictly defined and unambiguous terminology that promotes uniformity across users and institutions [14].

The risk stratification guidelines mentioned in this commentary are only a few of the many that have been developed worldwide over the last decade [15-17]. To address the uncertainty created by diverse algorithms, some of which result in conflicting management recommendations, the International Thyroid Nodule Ultrasound Working Group is undertaking a multidisciplinary effort to harmonize guidelines from multiple professional organizations (Franklin N. Tessler, MD, personal communication, Feb. 10, 2019). While this group's focus is on adults, the growing relevance of thyroid nodule ultrasound in pediatrics indicates that children, too, could benefit from 
similar consideration. Building upon an evolving understanding of the use of ultrasound guidelines in pediatrics, such initiatives can continue to help drive forward standardized clinical decision-making and improved care for children with thyroid nodules.

\section{Compliance with ethical standards}

Conflicts of interest None

\section{References}

1. Shapira-Zaltsberg G, Miller E, Martinez-Rios C et al (2019) Comparison of the diagnostic performance of the 2017 ACR TIRADS guideline to the Kwak guideline in children with thyroid nodules. Pediatr Radiol. https://doi.org/10.1007/s00247-01904385-6

2. Kwak J, Han K, Yoon J et al (2011) Thyroid imaging reporting and data system for US features of nodules: a step in establishing better stratification of cancer risk. Radiology 260:892-899

3. Tessler FN, Middleton WD, Grant EG et al (2017) ACR thyroid imaging, reporting and data system (TI-RADS): white paper of the ACR TI-RADS committee. J Am Coll Radiol 14:587-595

4. Middleton WD, Teefey SA, Reading CC et al (2017) Multiinstitutional analysis of thyroid nodule risk stratification using the American College of Radiology thyroid imaging reporting and data system. AJR Am J Roentgenol 208:1331-1341

5. Ha S, Ahn H, Baek JH et al (2017) Validation of three scoring riskstratification models for thyroid nodules. Thyroid 27:1550-1557

6. Lim-Dunham JE, Erdem Toslak I, Reiter MP, Martin B (2019) Assessment of the American College of Radiology thyroid imaging reporting and data system for thyroid nodule malignancy risk stratification in a pediatric poplulation. AJR Am J Roentgenol 212:1-7

7. Francis GL, Waguespack SG, Bauer AJ et al (2015) Management guidelines for children with thyroid nodules and differentiated thyroid cancer. Thyroid 25:716-759

8. Haugen B, Alexander EK, Bible KC et al (2016) 2015 American Thyroid Association management guidelines for adult patients with thyroid nodules and differentiated thyroid cancer. Thyroid 26:1412

9. Creo A, Alahdab F, Al Nofal A et al (2018) Ultrasonography and the American Thyroid Association ultrasound-based risk stratification tool: utility in pediatric and adolescent thyroid nodules. Horm Res Paediatr 90:93-101

10. Martinez-Rios C, Daneman A, Bajno L et al (2018) Utility of adultbased ultrasound malignancy risk stratifications in pediatric thyroid nodules. Pediatr Radiol 48:74-84

11. Lim-Dunham JE, Erdem Toslak I, Alsabban K et al (2017) Ultrasound risk stratification for malignancy using the 2015 American Thyroid Association management guidelines for children with thyroid nodules and differentiated thyroid cancer. Pediatr Radiol 47:429-436

12. Hoang JK, Middleton WD, Farjat AE et al (2018) Interobserver variability of sonographic features used in the American College of Radiology thyroid imaging reporting and data system. AJR Am J Roentgenol 211:1-6

13. Tessler FN, Middleton WD, Grant EG (2018) Thyroid imaging reporting and data system (TI-RADS): a user's guide. Radiology 287:29-36

14. Grant EG, Tessler FN, Hoang JK et al (2015) Thyroid ultrasound reporting lexicon: white paper of the ACR thyroid imaging, reporting and data system (TIRADS) committee. J Am Coll Radiol 12:1272-1279

15. Gharib H, Paschke R, Vitti P et al (2016) American Association of Clinical Endocrinologists, American College of Endocrinology, and Associazione Medici Endocrinologi medical guidelines for clinical practice for the diagnosis and management of thyroid nodules - 2016 update. Endocr Pract 22:622-639

16. Shin J, Baek J, Chung J et al (2016) Ultrasonography diagnosis and imaging-based management of thyroid nodules: revised Korean Society of Thyroid Radiology consensus statement and recommendations. Korean J Radiol 17:370-395

17. Russ G, Bonnema SJ, Erdogan MF et al (2017) European thyroid association guidelines for ultrasound malignancy risk stratification of thyroid nodules in adults: the EU-TIRADS. Eur Thyroid J 13: $225-237$

Publisher's note Springer Nature remains neutral with regard to jurisdictional claims in published maps and institutional affiliations. 